\title{
Vivid visual mental imagery in the absence of the primary visual cortex
}

\author{
Holly Bridge • Stephen Harrold • Emily A. Holmes • \\ Mark Stokes $\cdot$ Christopher Kennard
}

Received: 14 September 2011 / Accepted: 18 October 2011/Published online: 8 November 2011

(C) The Author(s) 2011. This article is published with open access at Springerlink.com

\begin{abstract}
The role of the primary visual cortex in visual mental imagery has provided significant debate in the imagery literature. Functional neuroimaging studies show considerable variation depending on task and technique. Patient studies can be difficult to interpret due to the diverse nature of cortical damage. The type of cortical damage in patient SBR is exceedingly rare as it is restricted to the gray matter of the calcarine sulcus. In this study, we show that in spite of his near-complete cortical blindness, SBR exhibits vivid visual mental imagery both behaviorally and when measured with functional magnetic resonance imaging. The pattern of cortical activation to visual mental imagery in SBR is indistinguishable from individual sighted subjects, in contrast to the visual perceptual responses, which are greatly attenuated.
\end{abstract}

Keywords Mental imagery - Cortical damage .

Primary visual cortex $\cdot$ Cortical blindness

Electronic supplementary material The online version of this article (doi:10.1007/s00415-011-6299-z) contains supplementary material, which is available to authorized users.

\section{H. Bridge $(\square) \cdot S$. Harrold}

FMRIB Centre, John Radcliffe Hospital,

University of Oxford, Oxford OX3 9DU, UK

e-mail: holly.bridge@clneuro.ox.ac.uk

\section{E. A. Holmes}

Department of Psychiatry, University of Oxford, Oxford, UK

M. Stokes

Department of Experimental Psychology, University of Oxford,

Oxford, UK

C. Kennard

Nuffield Department of Clinical Neurosciences,

University of Oxford, Oxford, UK

\section{Introduction}

Visual mental imagery is the ability to produce a visual image independently of the visual input to the retina. There is some phenomenological correspondence of mental imagery with the images perceived when visual stimuli are presented to the eyes, although mental images are generally (and not surprisingly) weaker. Since the introduction of functional magnetic resonance imaging (fMRI) to the battery of tools used to investigate the human brain, there have been many investigations into the neural basis of mental imagery. Several studies have found that visual imagery evokes activation in visual association cortex, such as occipitotemporal and occipitoparietal regions [1-6]. Additionally, regions outside of the occipital cortex in the frontal lobe appear to show non-specific activation during visual imagery [4], perhaps providing top-down feedback to the specific sensory representation that is appropriate for the mental image.

Patient studies suggest that there is a dissociation between visual imagery and visual perception. Berhmann et al. [7] and Servos and Goodale [8] described individual patients suffering from visual agnosia, in whom visual imagery appeared to be intact as measured with a battery of tests. Conversely, Moro et al. [9] described two patients with intact primary visual cortex (V1) and visual perception, in whom visual imagery was severely impaired. Similarly, Zeman et al. [10] describe a patient who suddenly lost the ability to spontaneously generate visual mental images in the absence of any visual loss.

One issue that remains controversial is the role of V1 in visual mental imagery. To what extent is V1 required for visual mental imagery? Activation of V1 is only found in a subset of mental imagery experiments, with some reporting no activation of V1 (see Kosslyn and Thompson [6] for a comprehensive review of the early literature). It has been shown that rTMS over V1 can interfere with imagery of 
oriented gratings [11], but targeting of V1 with TMS is difficult [12]. A potential reconciliation of the data is that the role of $\mathrm{V} 1$ depends on the type of imagery; precise, detailed imagery is more likely to need V1. Clearly the result of Moro et al. described above indicates that V1 is not sufficient for imagery. Only one study has reported a patient (HS) who was transiently cortically blind, but yet reported 'seeing' images which were presumably mental images [13]. However, since the lesion of the calcarine sulcus was not complete, and no functional imaging data are available, it may be that there was some spared cortex contributing to perception. Another patient-SBR - has a very specific lesion to V1 bilaterally, with only the very anterior tip intact, and no evidence of direct damage to the adjacent white matter [14]. This patient therefore provides a unique opportunity to determine the effect of V1 damage on visual mental imagery. In our previous fMRI study we have shown abnormal gray matter intensity in his $\mathrm{T} 1$-weighted scan, abnormal perfusion to $\mathrm{V} 1$ relative to area MT+ and a lack of BOLD activity in V1.

The contentious nature of investigating mental imagery is at least in part due to the lack of objective criteria. With no external stimulus, imagery is a classic subjective phenomenon. Attempts to address this inherent subjectivity have used measures of reaction time, and tasks that require using a mental image for performance, although this arguably just changes the problem. fMRI can provide a unique insight into imagery, without employing an additional task, such as rotation. However, the correlation between subjective reports of imagery strength and BOLD activity level is not straightforward. One study that found a correlation between the vividness of visual imagery questionnaire [15] and mental imagery used BOLD activity throughout the entire occipital lobe and relied on negative activity to drive the correlation [16]. Nonetheless, the presence of occipital cortical activation to visual mental imagery, combined with subjective reports of the images is strong evidence for successful imagery. While fMRI is a useful measure of activation, it does not provide any causal evidence. It is entirely possible that the activation of V1 in some imaging studies could reflect epiphenomenal activity. Therefore, this lesion fMRI study combines an objective measure of activation with causal inferences based on the absence of V1.

The nature of visual mental imagery in SBR was investigated using both fMRI and behavioral testing, and compared to eight young male control subjects.

\section{Methods}

\section{Subjects}

The study was conducted under ethical approval from the Oxfordshire NHS Research Ethics Committee B (08/
H0605/156) in accordance with the Declaration of Helsinki, and all subjects provided informed written consent. Eight young male control subjects were used for the experiment (mean age 20, range 17-25). All subjects had normal, or corrected-to-normal visual acuity. SBR has been described in detail in a previous paper (Bridge et al. 2010), and was 25 years old at the time of the current scans. He suffered a hypoxic event at the age of 22 , which led to a 3 -month period in a coma. On awaking, he was severely visually impaired, with no central vision present measured with perimetry. His visual fields, shown in Fig. 1, remain significantly reduced 3 years later.

\section{MR imaging}

Scanning was performed at the Oxford Centre for Functional Magnetic Resonance Imaging of the Brain (FMRIB) using a Siemens 3T Verio scanner with a 12-channel head coil. A $1 \times 1 \times 1 \mathrm{~mm}^{3} \mathrm{~T} 1$ structural scan was performed using standard parameters [magnetization prepared rapid gradient echo (MPRAGE), repetition time $(\mathrm{TR})=11.2$ $\mathrm{ms}$, echo time $(\mathrm{TE})=4.68 \mathrm{~ms}$.

Each subject participated in four functional MRI scans $(\mathrm{TR}=3 \mathrm{~s} ; 3.1 \mathrm{~mm} \times 3.1 \mathrm{~mm} \times 3 \mathrm{~mm} ; 44$ slices, $\mathrm{TE}=30 \mathrm{~ms}$ ). Scan one was the visual perception scan and consisted of $21 \mathrm{~s}$ blocks of either faces $\left(6.6^{\circ}\right.$ visual angle), houses $\left(6.6^{\circ}\right)$, flashing checkerboard $\left(7.4^{\circ} ; 8-\mathrm{Hz}\right.$ flicker) or a black fixation cross $\left(3^{\circ}\right)$ on a gray background. A new house or face stimulus was presented every second of the block. Each block was preceded and followed by a 1.5 -s period of fixation designed to match the period in which the auditory cue was given in the imagery scans. Blocks were presented four times each in pseudo-random order, determined by a Latin square design. Total scan length was therefore 6 min $24 \mathrm{~s}$. Scans 2-4 were imagery scans in which subjects were prompted with an auditory cue, to imagine either 'houses', 'faces' or 'chessboard' (1.5 s). The term 'chessboard' was employed as this is how most of the subjects described the checkerboard stimulus. After $21 \mathrm{~s}$, the subject heard the word 'stop' $(1.5 \mathrm{~s})$, before the next instruction was given. Again, blocks of different imagery instructions were presented in a pseudo-random order counterbalanced using a Latin square design. During the imagery scans, subjects were instructed to keep their eyes closed, and all lights were off in the scanner room.

\section{Data analysis}

Functional data were analyzed using FMRIB's easy analysis tool (FEAT), part of the FSL toolbox (www.fmrib. ox.ac.uk/fsl). For both imagery and visual perception scans, at a single scan level the images were pre-processed using a number of steps: head movement correction using 

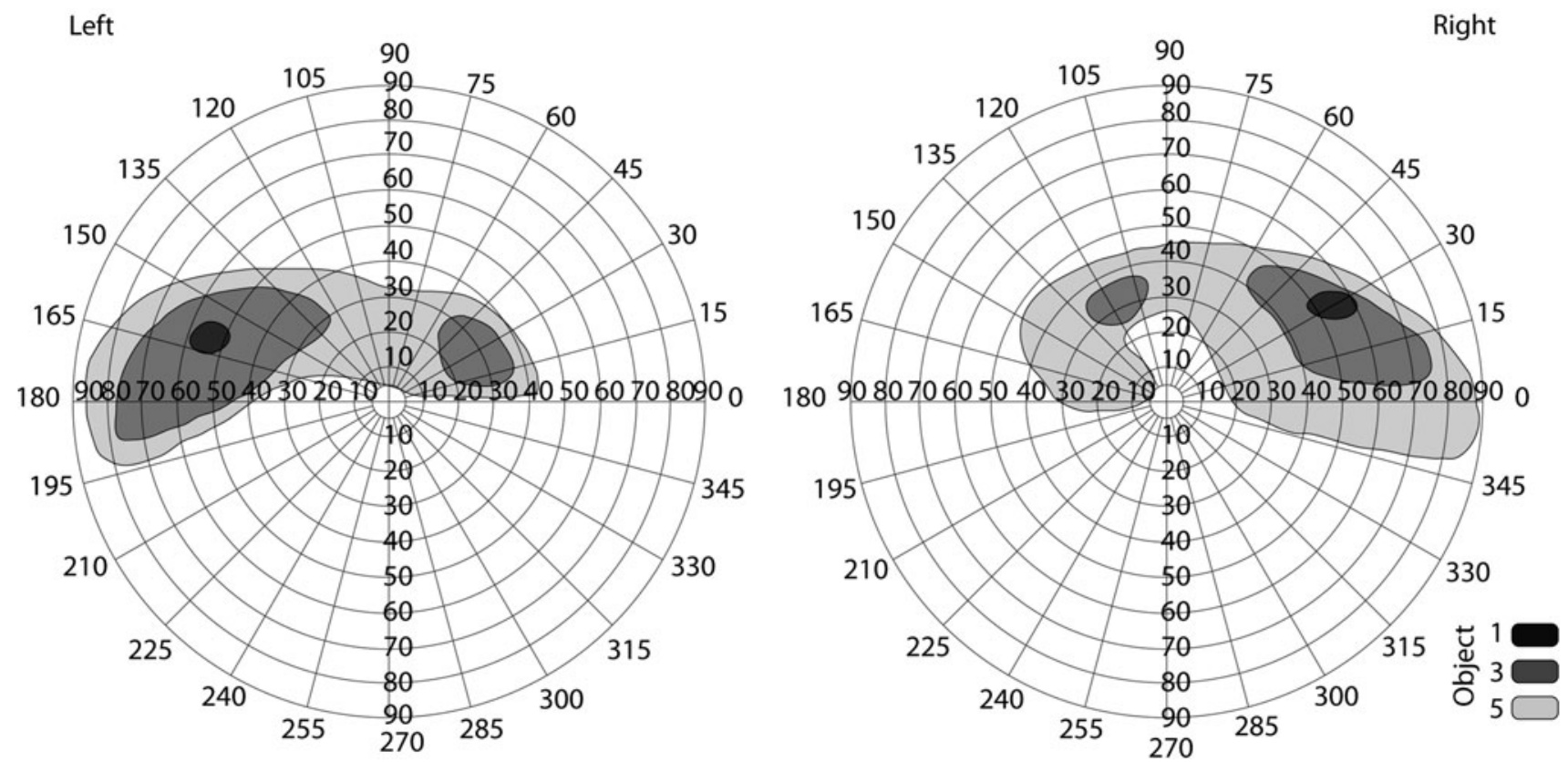

Fig. 1 Visual fields for SBR measured with Goldmann perimetry. He has no functional central vision, and can only perceive stimuli at the smallest size in the periphery

MCFLIRT, spatial smoothing with a Gaussian kernel full-width, half-height $(\mathrm{FWHM})=5 \mathrm{~mm}$, mean-based intensity normalization, non-linear high-pass temporal frequency filtering (Gaussian-weighted straight line fitting, with sigma $=30 \mathrm{~s}$ ). EPI data were registered to both the high-resolution MPRAGE structural scan and to the MNI152 standard brain using FLIRT, another tool from the FSL toolbox.

Single-scan (first level) analyses were combined across the three imagery scans for each subject using fixed-effects analysis. Group analyses (fixed effects) were then performed for each type of imagery (checkerboard, faces, houses). In the case of the visual perception scans, the first level analyses for each subject were fed into a group analysis (fixed effects) to produce the statistical maps. No thresholding was performed prior to the group analyses. The use of fixed effects implies that the results from the control group cannot be extrapolated to a general population. Mixed-effects analyses take into account intersubject variability, so tend to reduce the statistical significance of group activation. For the purpose of this study, however, it was more important to get an indication of the pattern of activation in the control subjects to compare with SBR, where there is no intersubject variability to take into account.

Segmentation, surface reconstruction, and inflation were performed on the structural scan of a control subject with the FreeSurfer software for visualization of the statistical activation data [17, 18]. Output from both the group analyses and the individual data from SBR was registered to the space of this brain in FreeSurfer using FLIRT. Other than the mapping to the cortical surface, no further analyses were performed in FreeSurfer.

The optimal method to define regions of interest to compare the levels of activation in SBR and sighted controls would be a localizer scan, but this is not possible for SBR. The primary visual cortex was defined using a probabilistic map defined using retinotopic mapping in 16 subjects [19]. This ROI for V1 was entirely within the damaged region of SBR, as the size of the retinotopic stimuli used for the mapping was well within his blind region. The fusiform face area was defined as a spherical ROI, radius of $6 \mathrm{~mm}$, centered upon the co-ordinates from the localizer in experiment one of Andrews et al. [20] (left $-38-61$ 18; right $41-55-25)$. Similarly, the parahippocampal place area was also defined as a spherical ROI, radius $6 \mathrm{~mm}$ centered upon the co-ordinates used by Park and Chun [21] (left $-27,-46,-15$; right 24, -33, -23). Additionally, a fourth (spherical; radius $8 \mathrm{~mm}$ ) medial visual area was defined at the intersection of the occipital, parietal, and temporal cortices, referred to here as the anterior calcarine, also known as retrosplenial cortex [22]. Percentage BOLD change was extracted from each of the imagery and visual scans in these four visual areas, resulting in a total of eight values for the control visual scans and 24 for the control imagery scans.

Analysis of variance was performed for each visual area to determine significant differences between stimulus type (faces, houses, checkerboards) and scan type (visual or imagery). 
Visual imagery testing

The vividness of visual imagery questionnaire (VVIQ) [23] was performed on each control subject and SBR to gain some idea of the individual abilities of the subjects to generate visual imagery. Additionally, SBR completed a short battery of imagery questionnaires (which could be administered aurally given that SBR was blind) administered by a clinical psychologist $(\mathrm{EH})$.

\section{Results}

Visual activation corresponds to expected regions of interest

The activation patterns to the visual stimuli consisting of faces, houses, and flickering checkerboard were as predicted for the control subjects. The largest responses to all stimuli were in V1, and then the fusiform face area (FFA). The parahippocampal place area showed the greatest activation to the house stimuli, but very little to the others. The most anterior portion of the calcarine showed more deactivation than activation in controls.

The devastating effect of SBR's damage to V1 can be seen by comparing the \% BOLD changes in this region with controls (see Fig. 2). All stimuli evoke a large response in V1 of the control subjects, with the greatest response to the flashing checkerboard. In contrast, none of the stimuli produce consistent activation in SBR, with no activation significantly greater than zero. Indeed, the response in SBR to checkerboards was more than two standard deviations away from the control group. The anatomically defined anterior calcarine region, which was previously shown to be intact in SBR, does show large responses. In particular, the checkerboard evokes large responses in this region in SBR, while being negatively activated in control subjects.

Although SBR claimed not to be able to perceive the visual stimuli, the faces and houses evoked patterns of activation similar to those in controls in the FFA, albeit at a lower level. There was also activation in SBR's PPA, although this was considerably more variable. SBR's activation to faces and houses are shown in Fig. 2b. The apparent lateralization to the right hemisphere indicates that SBR's eyes were probably not looking at the center of the screen.

Patterns of activation to mental imagery of houses, faces, and checkerboards

When asked to imagine houses, faces, or checkerboards, most control subjects were able to do this for the duration of the scan block. There was considerable variability, however, in the level and pattern of activation across subjects. The group activation is shown in Fig. 3 (lower row). The greatest activation is to the imagery of houses, where large regions of activation are present in both the anterior calcarine and the PPA. Mental imagery of faces also activates the anterior calcarine, and medial frontal regions. Despite the wide inter-subject variation in the cortical regions activated by the checkerboard condition, the fixed-effects analysis showed regions of parietal and lateral occipital cortex activated. No region of the medial occipital lobe was activated in this condition.

The equivalent patterns of activation in SBR differ according to the condition. In particular, the pattern of activation to imagery of houses is remarkably similar to the control group. The group data appear to be more reliable due to the type of analysis used. If a mixed-effects analysis is applied to the control data, the activation appears significantly lower than that in SBR (supplementary figure 1). Comparison of imagery of faces is also similar with the exception of the anterior calcarine, which is significantly activated in controls, but not at all in SBR. The reasons for this discrepancy are unclear, but presumably relate to the method used to generate the images.

The checkerboard appears to show the largest discrepancy between controls and SBR, the latter showing significant activity only in the fusiform region. In contrast, control subjects show a similar pattern of parietal and frontal activation as seen in the other conditions. However, there is considerable variability between control subjects, such that the use of a mixed-effects analysis results in minimal activation, as seen in supplementary figure 1 . The similarity between the data in SBR and the group, presented as \% BOLD change, is shown in Fig. 4. The pattern of activity across all the regions implicated in imagery is comparable for SBR and controls. Indeed, while SBR reported being unable to perceive the visual stimuli, he confidently reported being able to generate images of faces and houses, although he found checkerboards more difficult.

Interestingly, imagery of houses evoked a response just significantly greater than baseline in the primary visual cortex of control subjects $(t=2.0, d f=23, p<0.05)$. However, the similarity of activation to imagery of houses between controls and SBR outside of V1 is striking, suggesting that a lack of V1 activation does not affect activation in extrastriate areas.

In sighted subjects, occipital responses to perception are greater than to mental imagery

Comparison of responses to visual stimulation and visual mental imagery in each of the visual regions of interest 


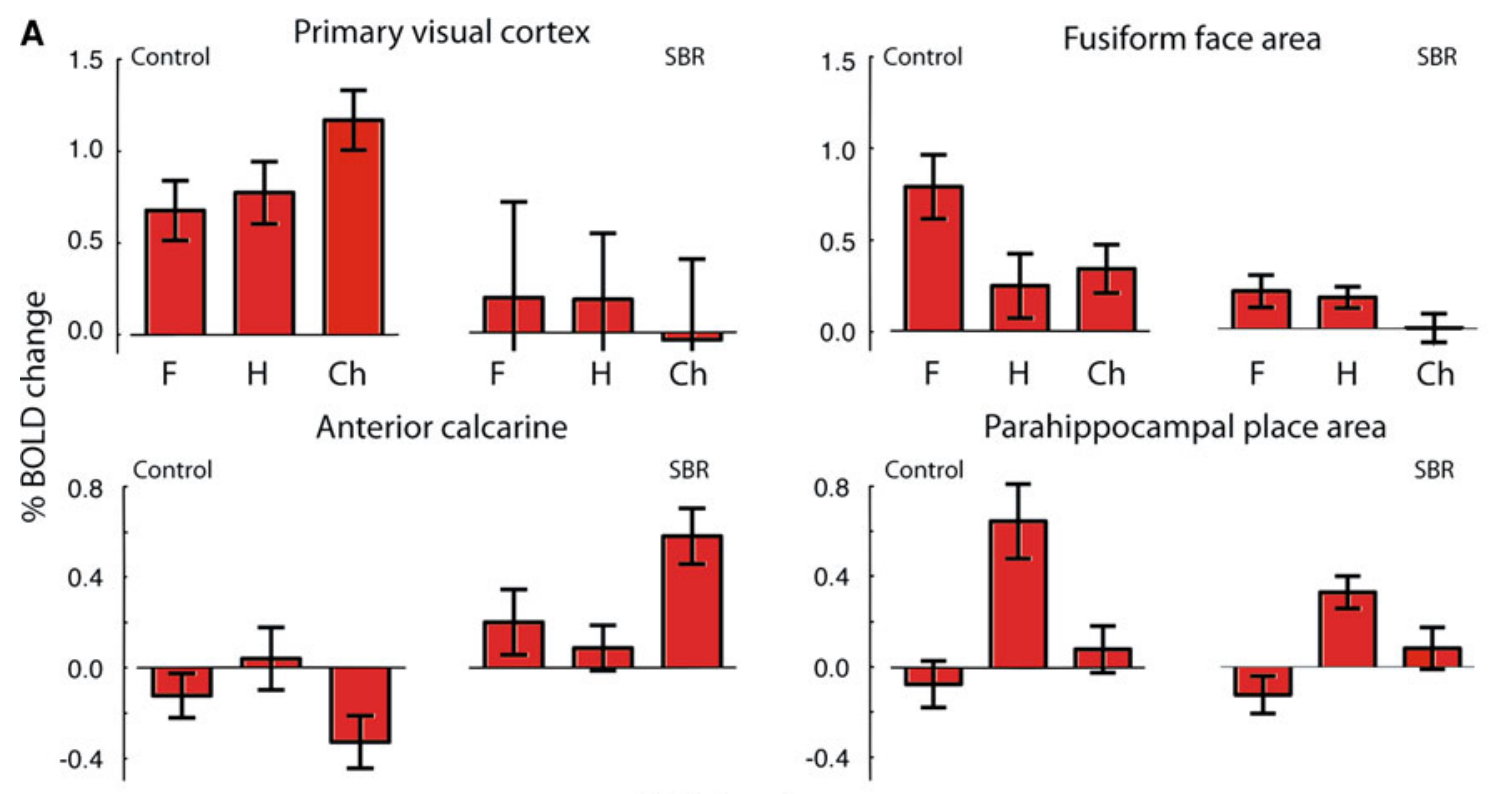

SBR visual responses

B

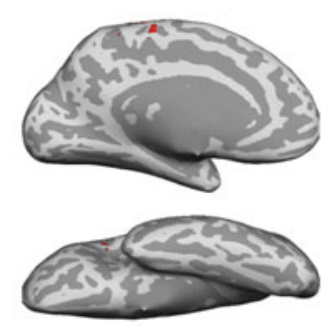

Faces

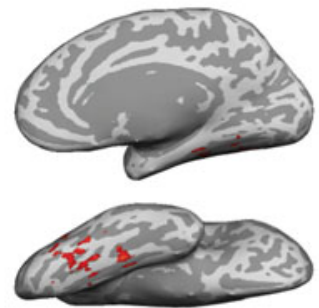

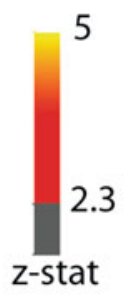

Houses
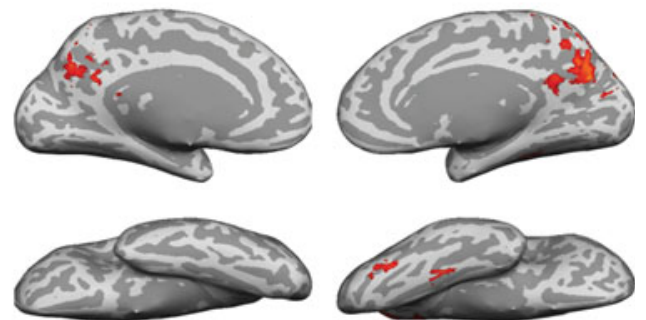

Checkerboard

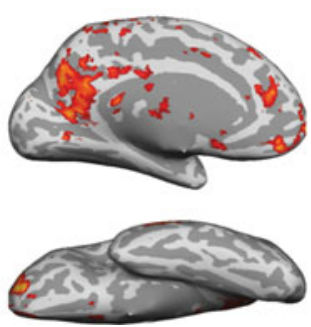

Fig. 2 a Responses of control subjects and SBR to visual stimulation. The bar charts show the \% BOLD change to faces, houses, and checkerboard compared to fixation across a range of visual cortical regions for the control group and for the blind subject SBR. Interestingly, while SBR shows the smallest response to the checkerboard in V1, it is this stimulus that evokes the greatest activation outside of V1. Error bars for control data show standard

showed significant differences. A two-way ANOVA showed a highly significant effect of stimulus type in V1 $(F=62.2 ; p<<0.001 ; d f=1)$ and FFA $(F=10.2$; $p<0.005 ; d f=1$ ) with greater responses to visual stimuli. In contrast, the significant effect of stimulus type in the anterior calcarine region reflected greater activation to

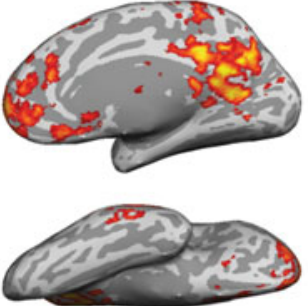

errors of the mean across the group. Error bars on SBR show withinscan variance calculated from the single scan run. b Shows the activation to visual stimuli in SBR's brain. The greater activity in the right hemisphere suggests that his eyes were not directed to the centre of the screen. Unlike control subjects in whom the greatest activation to the checkerboard stimulus is in the occipital lobe, the visual activity to the stimulus is spread throughout the cortex in SBR

mental imagery $(F=23.6 ; p<<0.001 ; d f=1)$. The PPA was the only region that showed no effect of stimulus type.

These results contrast sharply with the data from SBR in which the activation to imagery is greater than visual stimuli in both the FFA and the PPA. There are no responses significantly different from zero in V1 for either 


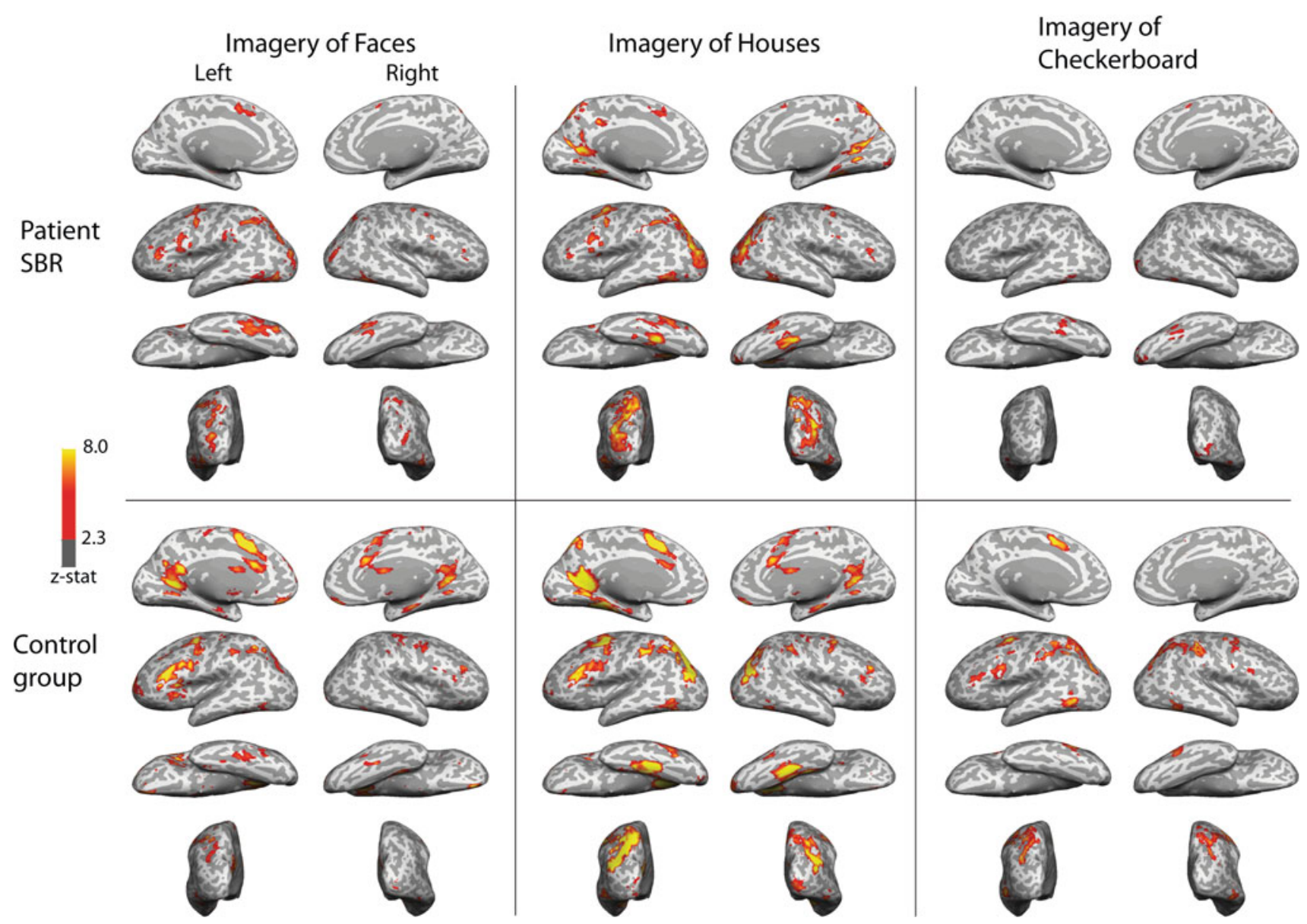

Fig. 3 Upper panel shows the activation of SBR's brain to imagery of faces, houses, and checkerboard. He shows extensive activation in the region of the intraparietal sulcus in addition to the fusiform gyrus for faces, and parahippocampal gyrus for faces. Additionally, further frontal regions are active in both conditions. In the case of the checkerboard, activation is limited to the fusiform region and lateral

stimulus type. In the anterior calcarine, imagery of houses evokes a considerably larger response than visual presentation, although visual presentation of both faces and checkerboards result in larger activation than imagery.

Behavioral measures of visual mental imagery

Most control subjects showed good levels of mental imagery vividness as reflected in the VVIQ, score mean of $2.3 \pm 0.6$. On this scale, 1 reflects extremely vivid images and 5 is unable to produce an image. SBR's vivid imagery was reflected in his score (1.9). In addition to his high score on the VVIQ, SBR shows high imagery performance across a battery of imagery questionnaires. In addition, he was able to readily describe detailed topographical imagery, e.g., the description of the route from his house to the pub; a detailed visual childhood image of playing in the garden describing colors, objects, and people; and a description of the shape of the Sydney Opera House (Table 1). region of the right occipital pole. The lower panel shows the group activation patterns to mental imagery of faces, houses and checkerboard. In all cases, the left hemisphere appears to have the greater response. The anterior calcarine and parahippocampal gyrus show the greatest activation in the imagery of houses. All statistics are clustercorrected for multiple comparisons $(z>2.3 ; p<0.05)$

\section{Discussion}

This study has shown that visual mental imagery can be evoked strongly and successfully even when V1 is compromised. V1 is therefore not necessary for this type of mental imagery. Rather, the generation of images of faces and houses appears to be mediated by activation of a network of extrastriate visual areas both in the dorsal and ventral stream.

Large-scale studies of brain-damaged patients appear to indicate that the greatest deficits in visual mental imagery occur following left posterior occipital damage [29]. A split-brain subject investigated by Farah et al. [30] was unable to perform visual mental imagery when the cue for the imagery was presented in the left visual field. This suggests that the right hemisphere was unable to support visual mental imagery. Furthermore, Stokes et al. [31] also found a left hemisphere bias in the lateral occipital cortex for mental imagery classification. These findings are 


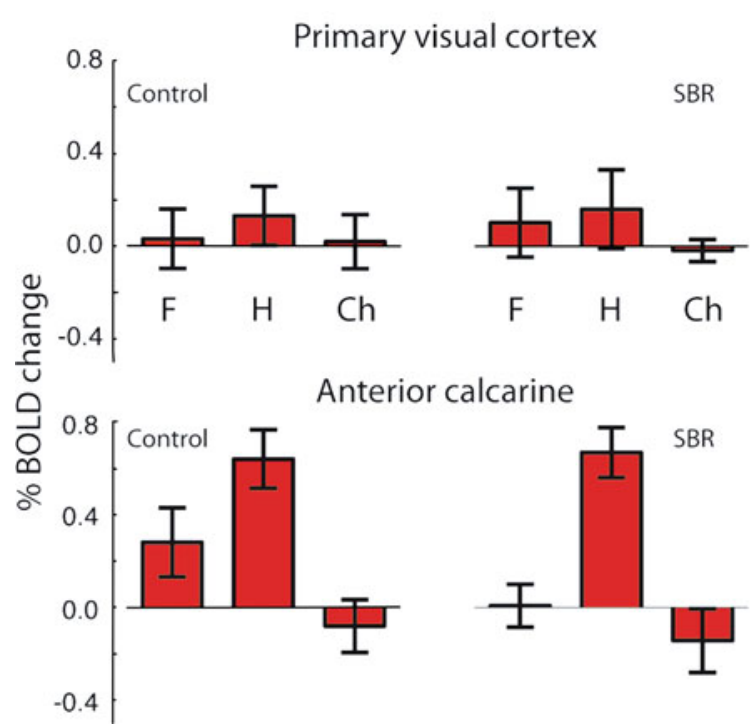

Fig. 4 The calculation of \% BOLD change evoked by visual mental imagery shows the similarity of response between the control subjects and blind subject SBR. Error bars show standard errors. In each case,
Fusiform face area

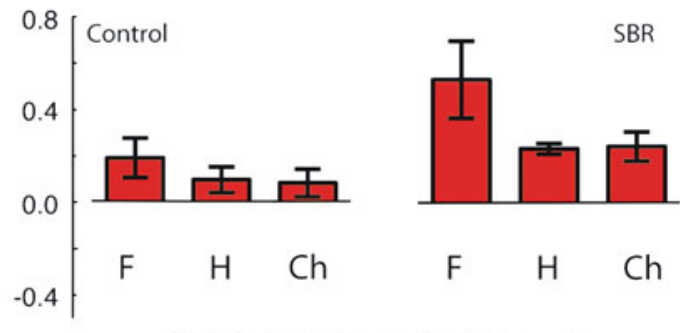

Parahippocampal place area

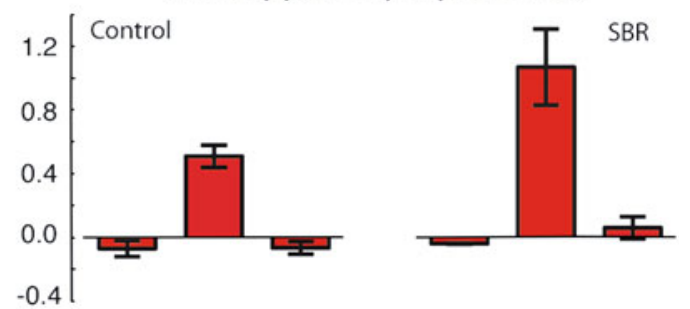

the left bar $(F)$ corresponds to faces, middle $(H)$ to houses and right $(\mathrm{Ch})$ is checkerboard
Table 1 SBR's performance on a battery of mental imagery questionnaires

Control subjects performed only the VVIQ

\begin{tabular}{ll}
\hline Test & Score \\
\hline Spontaneous use of imagery scale: (Reisberg et al. 2003) & 3.1 (control mean 3.1) \\
Controllability of visual imagery: Richardson [24]) & $24 / 24$ \\
Size comparison of paired animals task: Kosslyn et al. [25]; & $18 / 20$ \\
$\quad$ Policardi et al. [26] & \\
Straight/curves letter task: Coltheart et al. [27] & $20 / 20$ \\
Top/bottom larger letter task: Coltheart et al. [27] & $6 / 7$ \\
Mental hue comparison task: De Vreese [28] & $24 / 30$ \\
\hline
\end{tabular}

consistent with the greater activation to imagery in the left hemisphere in both SBR and the control group.

V1 has already been shown not to be sufficient for visual mental imagery. Moro et al. [32] have described two patients without damage to V1, both of whom show deficits in visual mental imagery, with one also showing deficits of tactile imagery. There was damage to left ventral occipital regions in both cases, with one also showing bilateral damage to parietal cortex. Again, this is consistent with the patterns of activation seen in controls and SBR in the current study.

The patterns of activation to imagery in SBR are very similar to those expected from visual perception. This strongly suggests that in spite of the almost complete absence of V1, extrastriate cortical areas are intact and can be significantly activated. It will be interesting to monitor the function of these extrastriate visual areas over time to determine whether they remain visual in a similar way to that reported in late blindness [33].

One point of potential interest is that SBR did not show much significant activation to the checkerboard. However, this was also the case for many of the control subjects. The group data showing \% signal change in Fig. 4 indicate the lower response in this imagery condition. Indeed, the intersubject variability can be seen in the BOLD responses of Fig. 4, but also in the finding that a mixed-effects analysis (which takes account of inter-subject variance) produced no significant activation within the occipital and parietal regions. The control subjects were also shown checkerboard stimuli in the visual stimulation experiment prior to the imagery scans, so they presumably had an advantage over SBR who had no recent accurate image of this stimulus.

It may be that it is particularly difficult to produce a mental image of such a basic stimulus. The extensive research by Kosslyn and his colleagues leads them to suggest that mental imagery requiring fine-grained spatial detail is likely to activate V1 [34]. In the meta-analysis of studies involving visual mental imagery by Kosslyn and Thompson [6] there is a list of studies that produced activation of V1 in control subjects. A limitation of using a patient such as SBR is that it is not possible to first present 
a visual stimulus and then request that the same, or a similar, mental image is produced. In spite of this limitation, two of the tasks reported to produce activation in V1, navigation around one's hometown [35] and imagery of animals [36] were successfully performed by SBR during interview. Additionally, SBR can generate 'basic' images such as black and white stripes of varying thickness and upper and lower case letters at different sizes.

The robust imagery experienced by SBR raises the question of whether V1 activation during visual mental imagery is an epiphenomenon not necessary for the experience of the image. One of the predictions in Kosslyn and Thompson is that damage that is specific to, and limited to V1 should produce retinotopic deficits in visual mental imagery. The presence of spared cortex at the very anterior section of SBR's calcarine cortex can address this prediction. The region corresponds visually to a spared temporal crescent around $40-50^{\circ}$ eccentric (as seen in Fig. 1), thus according to this requirement for intact V1 imagery should only be preserved in that region. In this case, however, the imagery spans the entire visual field, and many of the questionnaires performed on SBR require detailed analysis unlikely to be available so eccentrically in the visual field.

In conclusion, the data presented here show that when damage leading to cortical blindness is restricted to V1, visual mental imagery is intact and evokes widespread activation of extrastriate, parietal and frontal regions comparable to sighted subjects.

Acknowledgments We are grateful to head radiographer Stuart Wilson for his assistance in the smooth running of the project. We would like to thank SBR for his enthusiastic participation and generous offering of his time. This research was funded by the Royal Society (University Research Fellowship to HB), a Wellcome Trust Clinical Fellowship (WT088217 to EH) and the NIHR Biomedical Research Centre, Oxford (CK).

\section{Conflict of interest None.}

Open Access This article is distributed under the terms of the Creative Commons Attribution Noncommercial License which permits any noncommercial use, distribution, and reproduction in any medium, provided the original author(s) and source are credited.

\section{References}

1. Roland PE, Gulyas B (1994) Visual imagery and visual representation. Trends Neurosci 17(7):281-287 (discussion 294-287). pii:0166-2236(94)90057-4

2. Mellet E, Tzourio N, Crivello F, Joliot M, Denis M, Mazoyer B (1996) Functional anatomy of spatial mental imagery generated from verbal instructions. J Neurosci 16(20):6504-6512

3. Mellet E, Tzourio N, Denis M, Mazoyer B (1998) Cortical anatomy of mental imagery of concrete nouns based on their dictionary definition. Neuroreport 9(5):803-808
4. Ishai A, Ungerleider LG, Haxby JV (2000) Distributed neural systems for the generation of visual images. Neuron 28(3):979990

5. Amedi A, Malach R, Pascual-Leone A (2005) Negative BOLD differentiates visual imagery and perception. Neuron 48(5):859 872. doi:10.1016/j.neuron.2005.10.032

6. Kosslyn SM, Thompson WL (2003) When is early visual cortex activated during visual mental imagery? Psychol Bull 129(5):723-746. doi:10.1037/0033-2909.129.5.723

7. Behrmann M, Moscovitch M, Winocur G (1994) Intact visual imagery and impaired visual perception in a patient with visual agnosia. J Exp Psychol Hum Percept Perform 20(5):1068-1087

8. Servos P, Goodale MA (1995) Preserved visual imagery in visual form agnosia. Neuropsychologia 33(11):1383-1394

9. Moro V, Berlucchi G, Lerch J, Tomaiuolo F, Aglioti SM (2008) Selective deficit of mental visual imagery with intact primary visual cortex and visual perception. Cortex 44(2):109-118. doi: 10.1016/j.cortex.2006.06.004

10. Zeman AZ, Della Sala S, Torrens LA, Gountouna VE, McGonigle DJ, Logie RH (2010) Loss of imagery phenomenology with intact visuo-spatial task performance: a case of 'blind imagination'. Neuropsychologia 48(1):145-155. doi:10.1016/j.neuropsy chologia.2009.08.024

11. Kosslyn SM, Pascual-Leone A, Felician O, Camposano S, Keenan JP, Thompson WL, Ganis G, Sukel KE, Alpert NM (1999) The role of area 17 in visual imagery: convergent evidence from PET and rTMS. Science 284(5411):167-170

12. Thielscher A, Reichenbach A, Ugurbil K, Uludag K (2010) The cortical site of visual suppression by transcranial magnetic stimulation. Cereb Cortex 20(2):328-338. doi:10.1093/cercor/ bhp 102

13. Goldenberg G, Mullbacher W, Nowak A (1995) Imagery without perception - a case study of anosognosia for cortical blindness. Neuropsychologia 33(11):1373-1382

14. Bridge H, Hicks SL, Xie J, Okell TW, Mannan S, Alexander I, Cowey A, Kennard C (2010) Visual activation of extra-striate cortex in the absence of V1 activation. Neuropsychologia 48(14):4148-4154. doi:10.1016/j.neuropsychologia.2010.10.022

15. Marks DF (1973) Visual imagery differences in the recall of pictures. Br J Psychol 64(1):17-24

16. Cui X, Jeter CB, Yang D, Montague PR, Eagleman DM (2007) Vividness of mental imagery: individual variability can be measured objectively. Vis Res 47(4):474-478. doi:10.1016/j.visres. 2006.11.013

17. Fischl B, Sereno MI, Dale AM (1999) Cortical surface-based analysis II: inflation, flattening, and a surface-based coordinate system. NeuroImage 9(2):195-207. doi:10.1006/nimg.1998.0396

18. Dale AM, Fischl B, Sereno MI (1999) Cortical surface-based analysis I: segmentation and surface reconstruction. NeuroImage 9(2):179-194. doi:10.1006/nimg.1998.0395

19. Bridge $H$ (2011) Mapping the visual brain: how and why. Eye (Lond) 25(3):291-296. doi:10.1038/eye.2010.166

20. Andrews TJ, Davies-Thompson J, Kingstone A, Young AW (2010) Internal and external features of the face are represented holistically in face-selective regions of visual cortex. J Neuro Off J Soc Neurosci 30(9):3544-3552. doi:10.1523/JNEUROSCI. 4863-09.2010

21. Park S, Chun MM (2009) Different roles of the parahippocampal place area (PPA) and retrosplenial cortex (RSC) in panoramic scene perception. NeuroImage 47(4):1747-1756. doi:10.1016/j. neuroimage.2009.04.058

22. Vann SD, Aggleton JP, Maguire EA (2009) What does the retrosplenial cortex do? Nature reviews Neuroscience 10(11):792-802. doi:10.1038/nrn2733

23. Marks DF (1973) Visual imagery differences in the recall of pictures. Br J Psychol 64(1):17-24 
24. Richardson A (1994) Individual differences in imaging: their measurement. origins and consequences. Baywood Publishing Company, Amityville

25. Kosslyn SM, Holtzman JD, Farah MJ, Gazzaniga MS (1985) A computational analysis of mental image generation: evidence from functional dissociations in split-brain patients. J Exp Psychol Gen 114(3):311-341

26. Policardi E, Perani D, Zago S, Grassi F, Fazio F, Ladavas E (1996) Failure to evoke visual images in a case of long-lasting cortical blindness. Neurocase 2(5):381-394

27. Coltheart M, Hull E, Slater D (1975) Sex differences in imagery and reading. Nature 253(5491):438-440

28. De Vreese LP (1991) Two systems for colour-naming defects: verbal disconnection vs colour imagery disorder. Neuropsychologia 29(1):1-18

29. Stangalino C, Semenza C, Mondini S (1995) Generating visual mental images: deficit after brain damage. Neuropsychologia 33(11):1473-1483

30. Farah MJ, Gazzaniga MS, Holtzman JD, Kosslyn SM (1985) A left hemisphere basis for visual mental imagery? Neuropsychologia 23(1):115-118

31. Stokes M, Thompson R, Cusack R, Duncan J (2009) Top-down activation of shape-specific population codes in visual cortex during mental imagery. J Neurosci 29(5):1565-1572. doi: 10.1523/JNEUROSCI.4657-08.2009

32. Moro V, Berlucchi G, Lerch J, Tomaiuolo F, Aglioti SM (2008) Selective deficit of mental visual imagery with intact primary visual cortex and visual perception. Cortex; a journal devoted to the study of the nervous system and behavior 44(2):109-118. doi: 10.1016/j.cortex.2006.06.004

33. Goyal MS, Hansen PJ, Blakemore CB (2006) Tactile perception recruits functionally related visual areas in the late-blind. Neuroreport 17(13):1381-1384. doi:10.1097/01.wnr.0000227990. 23046.fe

34. Kosslyn SM, Ganis G, Thompson WL (2001) Neural foundations of imagery. Nat Rev Neurosci 2(9):635-642. doi:10.1038/ 35090055

35. Chen W, Kato T, Zhu XH, Ogawa S, Tank DW, Ugurbil K (1998) Human primary visual cortex and lateral geniculate nucleus activation during visual imagery. Neuroreport 9(16):3669-3674

36. Klein I, Paradis AL, Poline JB, Kosslyn SM, Le Bihan D (2000) Transient activity in the human calcarine cortex during visualmental imagery: an event-related fMRI study. J Cogn Neurosci 12(2):15-23. doi:10.1162/089892900564037 